\title{
An insight into chronic kidney disease (CKD) through an unbalanced angiogenesis
}

Elham Farsi

EMAN Research and Testing Laboratory, Department of Pharmacology, School of Pharmaceutical Sciences, Universiti Sains Malaysia, Penang, Malaysia

Please cite this article:

Elham Farsi. (2017). An insight into chronic kidney disease (CKD) through balanced angiogenesis, 1(1), pages 036-038.

Significance | Unbalanced angiogenesis in chronic kidney diseases.

\section{Graphical Abstract}

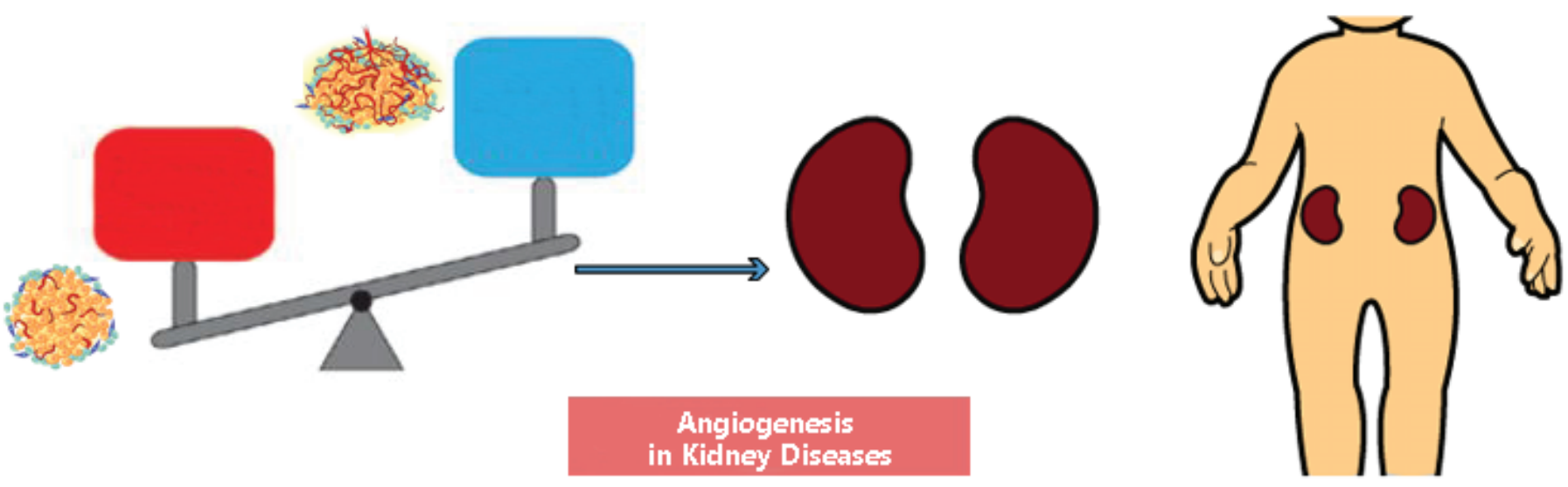

${ }^{*}$ Correspondence: EMAN Research and Testing Laboratory, Department of Pharmacology, School of Pharmaceutical Sciences, Universiti Sains Malaysia, Penang, Malaysia. E-mail: elhamfarsi@gmail.com 


\title{
An insight into chronic kidney disease (CKD) through an unbalanced angiogenesis
}

\author{
Elham Farsi
}

\section{Abstract}

Recent research data and supporting animal studies have shown that acute kidney injury (AKI) can eventually lead to chronic kidney disease (CKD). Moreover, the frequency of kidney transplants as result of CKD have been increasing daily. Various renal disorders result in end stage renal failure (ESRF) including glomerulonephritis, hypertensive nephrosclerosis and diabetic nephropathy (Levey and Coresh 2012). Chronic kidney disease involves conditions such as glomerulonephritis, glomerular endothelial cell injuries accompanies mesangial alterations or extracapillary lesions. To retrieve the glomerular endothelial cells in these models, angiogenesis stimulation is required to promote glomerular endothelial repair in association with accelerated resolution of nephritic alterations. The level of angiogenic signal should be adjusted according to the type and the stage of disease, to avoid an imbalance of pro / antigrowth and promotion of other pathologic condition as result of excessive angiogenesis (Goligorsky 2015; Levey and Coresh 2012). The development of new blood vessels, termed angiogenesis is responsible for several physiological and pathological events such as tumor growth and metastasis, proliferative retinopathy, rheumatoid arthritis, psoriasis and neointimal formation (Risau 1997). This process is controlled by pro and antigrowth factors and it is directly associated with the

Significance | Unbalanced angiogenesis in chronic kidney diseases.

${ }^{*}$ Correspondence: EMAN Research and Testing Laboratory, Department of Pharmacology, School of Pharmaceutical Sciences, Universiti Sains Malaysia, Penang, Malaysia. E-mail: elhamfarsi@gmail.com

Edited by Md Shamsuddin Sultan Khan, Hawkesbury Institute for the Environment, University of Western Sydney, Hawkesbury Campus, Bourke Street, Richmond, NSW AUSTRALIA 2753 and accepted by the Editorial Board May 8, 2017 (received for Sep 11, 2016) development of renal failure. Consequently, balanced ratios of these factors control severe renal conditions (Carmeliet 2003) The deregulated expression of factorsinhibitors ends in pathological conditions, like inflammation. Although findings about the biological role of angiogenesis factors in CKD are relatively limited, therapeutic effects have been reported for angiogenesis factors in experimental diabetic nephropathy models. Angiogenesis inhibitors exhibit therapeutic effects on diabetic nephropathy by anti-angiogenic and anti-inflammatory mechanisms. These effects highlight the importance of a tight regulation of angiogenic factors and inhibitors (Tanaka and Nangaku 2013).

Keywords: Chronic Kidney Disease, Angiogenesis

Abbreviations: VEGF, vascular endothelial growth factor

\section{Pro-angiogenesis factor in renal failure Vascular endothelial growth factor}

Vascular endothelial growth factor (VEGF) is an endothelial-specific growth factor that promotes endothelial cell proliferation, differentiation and survival, mediates endothelium-dependent vasodilatation, induces microvascular hyper-permeability and participates in interstitial matrix remodeling (Carmeliet 2003). The role of VEGF is unknown in normal kidney physiology, however VEGF and its receptor are up-regulated in type 1 and 2 diabetes. Also inhibition of VEGF and VEGFR expression ameliorate diabetic kidney condition (Schrijvers et al. 2004). VEGF plays an important role in maintaining the glomerular capillary struc-

Author Affiliation:

EMAN Research and Testing Laboratory, Department of Pharmacology, School of Pharmaceutical Sciences, Universiti Sains Malaysia, Penang, Malaysia

Please cite this article:

Elham Farsi. (2017). An insight into chronic kidney disease (CKD) through balanced angiogenesis , 1(1), pages 036-038. 
ture, and in the repair process following injuries to the glomerular endothelial cells and peritubular capillaries (PTC). Physiological levels of VEGF-A are also required for maintenance of glomerular filtration (Khamaisi et al. 2003).

\section{Angiopoietins}

Reduction in circulating levels of Ang-1, and elevation of Ang-2 levels in patients with CKD displays moderate to severe renal dysfunction. In a mouse model of folic acid-induced nephrotoxicity, Ang-1 was detected in renal arterial walls and in injured cortical distal tubules. In a mouse anti-glomerulonephritis model, the glomerular level of Ang-1 was reduced and Ang-2 was increased, in association with glomerular endothelial cell apoptosis. In a mouse model of unilateral ureteral obstruction, reduction in renal Ang-1 level was observed. This research data suggest that Ang-1 supplementation can have variable outcomes on renal injuries, depending on the disease model (Futrakul and Futrakul 2011; Woolf et al. 2009).

\section{Other angiogenic factors}

In a mouse model of ischemia-reperfusion injury (IRI), renal expression of Ephrin type-A receptor 2 (EphA2) mRNA was markedly increased and EphA2 protein was detected in distal tubules. The interactions between EphA2 and its Ephrin ligands may be involved in cytoskeletal repair of tubular epithelial cells in renal IRI. In the early stages ( 1 or 2 weeks) of the rat remnant kidney model, renal levels of Placental Growth Factor (PlGF) were elevated in association with enhanced angiogenic response. Whether VEGF-A or PlGF play principal roles in the angiogenic repair process after injuries induced by renal mass ablation needs further investigation.

\section{Role of angiogenesis inhibitors in CKD Angiostatin}

Angiostatin, a proteolytic fragment of plasminogen, potently blocks neovascularization, tumor growth and metastasis. In healthy rat kidneys, angiostatin is not expressed, but its level was enhanced in the first 3 days after renal ischemia-reperfusion injury (IRI) and remained detectable until 35 days after induction of IRI. Renal angiostatin generation after acute renal failure induced by IRI may modulate post-ischemic renal capillary density and thereby influence chronic renal dysfunction. Therefore, Angiostatin may exert therapeutic effects in CKD through its anti-inflammatory actions (Mu et al. 2009).

\section{Tumstatin}

Tumstatin peptide is an angiogenesis inhibitor derived from type IV collagen and inhibits in vivo neovascularization induced by vascular endothelial growth factor (VEGF), therefore inhibits angiogenesis and tumor growth by suppression of endothelial cell proliferation and tube formation (Noiri and Fujita 2010). One of the mediators of glomerular hypertrophy in diabetic nephropathy, glomerularhypertrophy, hyperfiltration, and albuminuria were suppressed by tumstatin peptide $(1 \mathrm{mg} / \mathrm{kg})$ in streptozotocin-induced diabetic mice. Tumstatin peptide, an inhibitor of angiogenesis, prevents glomerular hypertrophy in the early stage of diabetic nephropathy (Yamamoto et al. 2004).

\section{Nephrin}

Nephrin is a podocyte protein that is crucial for maintenance of an intact glomerular filtration barrier. In diabetic nephropathy, the protein level of Nephrin decreases in association with podocyte injury and proteinuria, and Tumstatin restored the level of Nephrin, possibly associated with its anti-albuminuric effects. The indirect influence of glomerular endothelial cells toward podocytes possibly mediated via secreted factors or alteration on matrix microenvironment might be involved in this mechanism.

\section{Endostatin}

Endostatin peptide, a potent inhibitor of angiogenesis derived from type XVIII collagen, is a potent preventer of type 1 diabetic nephropathy mouse model. The Endostatin peptide significantly suppressed the STZ-induced increase in VEGF-A, VEGFR-2, and Ang-2, and monocyte chemotactic protein (MCP)-1 in renal cortex and restored the level of Nephrin. Therefore, we speculate that Endostatin indirectly affects podocytes leading to decreased expression of VEGF-A in diabetic mice. Collectively, these results suggest that the Tumstatin peptide may have therapeutic potential in human diabetic nephropathy, in addition to its potent anti-tumor effect (Chen et al. 2012).

\section{Angiostatin}

Angiostatin is a proteolytic fragment of plasminogen and a potent angiogenic inhibitor. Previous studies have shown that angiostatin inhibits retinal neovascularization and reduces retinal vascular permeability in diabetic retinopathy (Chen et al. 2012). The potential involvement of angiostatin in diabetic nephropathy using an STZ-induced type 1 diabetes rat model was examined. Angiostatin levels were dramatically decreased in the kidneys of diabetic control rats, and adenovirus-mediated delivery of angiostatin significantly ameliorated albuminuria and glomerular hypertrophy in diabetic rats. Inhibitory effects of Angiostatin treatment on VEGF-A and TGF- $\beta 1$ in diabetic kidneys were also observed. Angiostatin produced direct effects on mesangial cells and endothelial cells, as well as therapeutic effects on diabetic nephropathy (Boor and Floege 2011; Zhang et al. 2006).

\section{Vasohibin-1}

Vasohibin (VASH)-1, an endogenous angiogenesis inhibitor, was identified in a microarray analysis, assessing genes upregulated by VEGF-A in endothelial cells. VASH-1 serves as an endothelial cell-derived negative feedback regulator of angiogenesis, and its therapeutic efficacies on tumor growth, atherosclerosis and proliferative retinopathy models have been reported. The studies on STZ-diabetic rats suggest therapeutic potential for VASH-1 in treating early diabetic nephropathy thought to be mediated via gl- 
omerular endothelial and mesangial cells (Maeshima and Makino 2010).

\section{Conclusion}

The finding revealed that advances study on the involvement of angiogenic and antiangiogenic factors in CKD and potential experimental approaches using angiogenesis inhibitors to treat CKD, including diabetic nephropathy. Based on the mechanistic insight into the involvement of angiogenesis in $\mathrm{CKD}$, the use of novel therapeutic reagents in patients with CKD in clinical practice is expected in the near future, leading to the eventual decrease in the number of patients developing ESRD.

\section{Acknowledgment}

The author would like to express his gratitude to his editorial fellows.

\section{Author Contribution}

Elham F. made substantial contributions to the conception of the review.

\section{Competing financial interests}

The author(s) declare no competing financial interests.

\section{References}

Boor, P. and Floege, J. (2011). Chronic kidney disease growth factors in renal

fibrosis. Clinical and Experimental Pharmacology and Physiology 38, 441-450.

Carmeliet, P. 2003. Angiogenesis in health and disease. Nature medicine 9, 653-660. Chen, J., Hamm, L.L., Kleinpeter, M.A., Husserl, F., Khan, I.E., Chen, C.-S., Liu, Y., Mills K.T., He, C. and Rifai, N. (2012). Elevated plasma levels of endostatin are associated with chronic kidney disease. American journal of nephrology 35, 335-340.

Futrakul, N. and Futrakul, P. (2011). Vascular homeostasis and angiogenesis determine therapeutic effectiveness in type 2 diabetes. International journal of vascular medicine 2011.

Goligorsky, M.S. (2015). Chapter 15 - Chronic Kidney Disease and Vascular Endothelium. In: P.L. Kimmel and M.E. Rosenberg (Eds), Chronic Renal Disease, Academic Press, San Diego, pp. 170-180

Khamaisi, M., Schrijvers, B.F., De Vriese, A.S., Raz, I. and Flyvbjerg, A. (2003). The emerging role of VEGF in diabetic kidney disease. Nephrology Dialysis Transplantation $18,1427-1430$

Levey, A.S. and Coresh, J. (2012). Chronic kidney disease. The Lancet 379, 165-180. Maeshima, Y. and Makino, H. (2010). Angiogenesis and chronic kidney disease. Fibrogenesis Tissue Repair 3, 1-17

Mu, W., Long, D.A., Ouyang, X., Agarwal, A., Cruz, P.E., Roncal, C.A., Nakagawa, T., Yu, X., Hauswirth, W.W. and Johnson, R.J. (2009). Angiostatin overexpression is associated with an improvement in chronic kidney injury by an anti-inflammatory mechanism. American Journal of Physiology-Renal Physiology 296, F145-F152.

Noiri, E. and Fujita, T. (2010). Role of vascular endothelial growth factor in kidney disease. Current vascular pharmacology 8, 122-128.

Risau, W. (1997). Mechanisms of angiogenesis. Nature 386, 671-674.

Schrijvers, B.F., Flyvbjerg, A. and De Vriese, A.S. (2004). The role of vascular endothelial growth factor (VEGF) in renal pathophysiology. Kidney Int 65, 2003-2017.

Tanaka, T. and Nangaku, M. (2013). Angiogenesis and hypoxia in the kidney. Nature Reviews Nephrology 9, 211-222.

Woolf, A.S., Gnudi, L. and Long, D.A. (2009). Roles of angiopoietins in kidney develop- ment and disease. Journal of the American Society of Nephrology 20, 239-244.

Yamamoto, Y., Maeshima, Y., Kitayama, H., Kitamura, S., Takazawa, Y., Sugiyama, H., Yamasaki, Y. and Makino, H. (2004). Tumstatin peptide, an inhibitor of angiogenesis, prevents glomerular hypertrophy in the early stage of diabetic nephropathy. Diabetes 53, 1831-1840.

Zhang, S.X., Wang, J.J., Lu, K., Mott, R., Longeras, R. and Ma, J. (2006). Therapeutic potential of angiostatin in diabetic nephropathy. Journal of the American Society of Nephrology 17, 475-486.
Submit your next manuscript to Angiotherpay published by THE EMAN RESEARCH LTD.

- Convenient online submission

- Thorough peer review

- No space constraints or color figure charges

- Immediate publication on acceptance

- Inclusion in Australian National Libraray and Google Scholar

- Both Open (80-100\% subsidized APC by ER) \& non-open access option

Submit your manuscript at

angiotherapy.emanresearch.org 